Cahiers $d u$ MONDE RUSSE

\section{Cahiers du monde russe}

Russie - Empire russe - Union soviétique et États indépendants

48/2-3 | 2007

Les résonances de 1905

\title{
La réforme du ministère des affaires étrangères après la révolution de 1905
}

\section{Marie-Ève Rakuzin}

\section{OpenEdition}

\section{Journals}

Édition électronique

URL : https://journals.openedition.org/monderusse/9008

DOI : 10.4000/monderusse.9008

ISSN : $1777-5388$

\section{Éditeur}

Éditions de l'EHESS

\section{Édition imprimée}

Date de publication : 15 avril 2007

Pagination : 347-354

ISBN : 978-2-7132-2147-7

ISSN : $1252-6576$

Référence électronique

Marie-Ėve Rakuzin, "La réforme du ministère des affaires étrangères après la révolution de 1905 », Cahiers du monde russe [En ligne], 48/2-3 | 2007, mis en ligne le 01 janvier 2007, consulté le 04 septembre 2022. URL : http://journals.openedition.org/monderusse/9008 ; DOI : https://doi.org/ 10.4000/monderusse. 9008 
chercher : repérer : avancer

Cet article est disponible en ligne à l'adresse :

http://www.cairn.info/article.php?ID REVUE=CMR\&ID NUMPUBLIE=CMR 482\&ID ARTICLE=CMR 4820347

\title{
La réforme du ministère des affaires étrangères après la révolution de 1905
}

\author{
par Marie-Ève RAKUZIN
}

\section{Editions de l'EHESS | Cahiers du monde russe}

\author{
$2007 / 2-3-$ Vol 48 \\ ISSN 1252-6576 | ISBN 9782713221477 | pages 347 à 354
}

Pour citer cet article:

-Rakuzin M.-n, La réforme du ministère des affaires ét rangères après la révolution de 1905, Cahiers du monde russe 2007/ 2-3, Vol 48, p. 347-354.

Distribution électronique Cairn pour les Editions de l'EHESS.

(C) Editions de l'EHESS. Tous droits réservés pour tous pays.

La reproduction ou représentation de cet article, notamment par photocopie, n'est autorisée que dans les limites des conditions générales d'utilisation du site ou, le cas échéant, des conditions générales de la licence souscrite par votre établissement. Toute autre reproduction ou représentation, en tout ou partie, sous quelque forme et de quelque manière que ce soit, est interdite sauf accord préalable et écrit de l'éditeur, en dehors des cas prévus par la législation en vigueur en France. Il est précisé que son stockage dans une base de données est également interdit. 


\section{LA RÉFORME DU MINISTÈRE DES AFFAIRES ÉTRANGÈRES APRÈS LA RÉVOLUTION DE 1905}

Les événements de 1904-1905 ont accéléré un mouvement qui était déjà en gestation depuis la fin du XIX siècle. Les prémisses de la réforme du ministère des Affaires étrangères (Ministerstvo inostrannyh del - MID), qui fut mise en place officiellement sous l'impulsion de Aleksandr Petrovič Izvol'skij en 1907, remontent aux années 1895-1896, sous le ministère du prince Lobanov-Rostovskij. À son arrivée il tenta, de réformer le MID, en confiant à F.F. Martens (1845-1909), spécialiste russe en droit international et membre permanent du Conseil du ministère, la tâche d'exposer très clairement les raisons d'une telle réforme. À la fin de l'année 1895, Martens présenta une note concernant la nécessité d'une réorganisation du MID (Zapiska o preobrazovanii MID)1 ${ }^{1}$, dans laquelle il proposait une nouvelle structure du ministère et une fusion de tous ses départements politiques en une seule section, comme cela se pratiquait dans les ministères des Puissances européennes. Dans une lettre adressée au prince Lobanov-Rostovskij, il expliquait le besoin pressant d'en finir avec l'indépendance des directeurs des départements qui menaient chacun une politique totalement indépendante de celle qui était prônée par le ministre des Affaires étrangères. Auparavant, il s'était expliqué sur la nécessité d'une telle réforme, en invoquant le fait qu'un pays ne pouvait pas fractionner sa politique extérieure en plaçant les affaires d'Orient dans un Département asiatique, et celles relatives aux pays européens dans la Chancellerie du ministère.

Aux événements de la révolution de 1905 est venue s'ajouter la défaite de la Russie face au Japon la même année. L'expansion russe en Extrême-Orient,

\footnotetext{
* Cet article s'inscrit dans une recherche plus large qui a débouché sur une thèse de doctorat, soutenue en 2003 et intitulée «Les diplomates russes entre 1880 et 1914 : perception identitaire, activité professionnelle et réflexions politiques ».

1. AVPRI (Arhiv vnešnej politiki Rossijskoj imperii - Archives de la politique étrangère de l'Empire russe), f. 159 , op. 731 , d. 28, 1. 4-17. Projet de réforme du ministère 1895-1896; 1906.
} 
commencée encore dans les années 1890, fut stoppée par le Japon en 1904 par une attaque contre la Russie ${ }^{2}$. Depuis la fin du XIX ${ }^{\mathrm{e}}$ siècle, les deux pays se disputaient des possessions en Corée et en Mandchourie. La défaite russe, sanctionnée par le traité de Portsmouth en septembre 1905, marqua de manière durable la politique extérieure et intérieure de la Russie, et ce jusqu'en 1914³. La défaite face au Japon accrut la nécessité de changement du mode de fonctionnement du ministère et la révolution de 1905 sonna l'alerte pour une réforme du gouvernement dans son ensemble, et celui du ministère des Affaires étrangères en particulier.

À partir de 1904, après l'instauration d'un «second ministère des Affaires étrangères » chargé des questions de l'Extrême-Orient et du déclenchement de la guerre avec le Japon, le ton des lettres des diplomates change. Ceci est particulièrement sensible dans les lettres du comte Aleksandr Hristoforovič Benkendorf, ambassadeur extraordinaire et ministre plénipotentiaire à Londres depuis 1902, qui souligne la nécessité de prendre part à l'action politique du pays.

Dans une lettre adressée au comte Lamzdorf (alors ministre des Affaires étrangères), datée de la fin de l'année 1904, Benkendorf voyait dans le système structurel du gouvernement lui-même la cause du manque d'autorité du ministère des Affaires étrangères en matière de politique étrangère :

C'est toujours le même système. Des improvisateurs monopolisent le patriotisme, présentent les cas les plus difficiles comme tout simples, parlent de choses qu'ils ne connaissent pas, enflamment le public, l'induisent dans la plus déplorable des erreurs et des illusions, et si tant est qu'ils pensent aux obstacles insurmontables qui peuvent surgir sur la route de leurs projets, ou les abandonner charitablement au ministère des Affaires étrangères, quitte à celui-ci à se tirer de là comme il peut, et quand la situation qu'on lui donne à refaire est irrefaisable, c'est le ministère des Affaires étrangères qui a tort et est condamné. Intentionnel ou non, je ne connais rien de plus déloyal, de plus léger et de plus coupable envers la Russie.

Quand tout ce déplorable travail et état des choses finira-t-il ? Les temps sont favorables pour les brouillons irresponsables. Et j'avoue que cela frappe spécialement ici, où rien de pareil ne paraît possible que face à face avec un ministre prêt à $\underline{\text { répondre. }}$. Comment vraiment ne pas comprendre encore que c'est grâce à ce système de désorganisation et pour avoir ignoré comme à dessein toutes les difficultés, que nous avons cette néfaste guerre. ${ }^{4}$

2. Voir à ce sujet, David Walder, The Short Victorious War. The Russo-Japanese Conflict 1904-1905, Londres: Hutchinson, 1973 ; Boris Aleksandrovič Romanov, Očerki diplomatičeskoj istorii russko-japonskoj vojny, 1895-1907 [Histoire diplomatique de la guerre russo-japonaise, 1895-1907], 2e édition, M.-L.: Izdat. Akademii nauk SSSR, 1955.

3. Anatolij Venediktovič Ignat'ev, Vnešnjaja politika Rossii 1905-1907[La politique étrangère de la Russie, 1905-1907], M.: Nauka, 1986 ; Vnešnjaja politika Rossii, 1907-1914. Tendencii, ljudi, sobytija [La politique étrangère de la Russie, 1907-1914. Tendances, populations, événements], M: Nauka, 2000 ; «Politika soglašenija i balansirovanija (Vnešnepolitičeskij kurs Rossii v 1906-1914 gg) [ «Politique de l'accord et de l'équilibre (Politique étrangère de la Russie 1906-1914)], Otečestvennaja istorija, n³ 3, 1997, p. 23-32.

4. AVPRI, f. 138, op. 467, d;225/226, 1.125. Lettre du comte Benkendorf au comte Lamzdorf, Londres, 28 novembre 1904. En français dans le texte. Expressions soulignées par l'auteur. 
Le comte Benkendorf mettait en lumière la difficulté que le ministère des Affaires étrangères avait à faire prévaloir ses visions politiques auprès du tsar : c'est en effet le tsar qui décidait en dernier lieu de la politique étrangère et devait donc répondre d'elle. Benkendorf faisait référence à la mise en place en 1903 d'un service totalement indépendant du ministère en Extrême-Orient, que le tsar confia au général Alekseev, soutenu par Bezobrazov. Il avait été créé dans un but politique et économique, mais était totalement soustrait de l'autorité du MID et de son ministre.

La première cause de la défaite russe face au Japon résultait de l'absence de coordination de travail entre les différents ministères, leurs ministres et le manque de responsabilité du ministère des Affaires étrangères face à ses choix politiques et à la manière de conduire la politique étrangère. Le comte se référait au système gouvernemental de la Russie, l'opposant au système constitutionnel instauré en Angleterre. Ces mots furent écrits fin 1904, au début des mouvements révolutionnaires qui amenèrent l'instauration d'un régime monarchique parlementaire. En effet, dès la fin du mois de novembre 1904, le mouvement des zemstva réclamait avec insistance un organe national représentatif pour restreindre ou contrôler les structures du gouvernement qu'il jugeait inefficace. Le parallèle que trace Benkendorf avec la Grande-Bretagne se comprend pour deux raisons : d'une part, il était en poste à Londres depuis 1902 et d'autre part, le système politique et social anglais jouissait d'une grande popularité dans les milieux de la noblesse russe. Le fait de perdre une partie du contrôle de la politique étrangère était inconcevable en Angleterre, puisque le ministre des Affaires étrangères, soutenu par le Premier ministre, avait à répondre de la politique de son gouvernement. Par ailleurs, ce modèle ne venait en rien remettre en cause la loyauté de Benkendorf envers la monarchie ${ }^{5}$. C'est la position du ministre au sein du gouvernement russe qui était directement visée: il fallait faire participer le ministre à la vie du gouvernement et à sa politique. Il fallait lui accorder une entière autonomie et indépendance de vue dans ses prises de position, mais dans le cadre d'un gouvernement uni. En Russie, le ministre des Affaires étrangères était un exécutant de la volonté du tsar, mais devait aussi répondre de la politique étrangère, décidée non par lui mais par le monarque, lui conférant ainsi une «certaine» responsabilité dans la poursuite de la politique étrangère que par ailleurs il ne détenait pas. Le comte sous-entendait une réforme en profondeur du système politique russe, dans lequel les rôles et les devoirs auraient dû être clairement établis. Benkendorf touchait au problème central de l'autocratie et à la réglementation de ses pouvoirs.

La Russie avait déjà été confrontée à ce genre de prises de position. Après la guerre de Crimée, certains ministres, dont celui des Affaires intérieures, Petr Aleksandrovič Valuev, soutenu par de hauts fonctionnaires, - parmi lesquels le comte Petr Andreevič Šuvalov - tentèrent de réorganiser le système gouvernemental russe et d'instaurer ce qu'ils appelèrent un ob"edinennoe pravitel'stvo

5. J. Armstrong, « Mobilized Diaspora in Tsarist Russia : the Case of the Baltic Germans », in Jeremy R. Azrael, ed., Soviet Nationality Policies and Practices, New-York :Praeger, 1978, p. 90 . 
[gouvernement d'union] ${ }^{6}$. Ce gouvernement ${ }^{7}$ inspiré par le modèle constitutionnaliste anglais maintenait l'autocratie, mais dans un cadre très restrictif : le tsar se voyait dépouillé d'une partie de ses prérogatives politiques par l'introduction d'un Cabinet ministériel (le terme est employé à cette période), mais, à l'époque, conservait celles relatives à la politique étrangère. Le comte Benkendorf, sans le moindre doute, réintroduisit ce débat dans ses lettres : en effet, en plus d'être anglophile, il était parent par alliance de Šuvalov. Par ailleurs, ces idées étaient bien connues au sein du ministère des Affaires étrangères, dont certains des hauts fonctionnaires avaient participé à ces débats dans les années 1870, comme le prince A.B. Lobanov-Rostovskij ${ }^{8}$, ou étaient connus comme sympathisants aux idées constitutionnelles promues dans les années 1870 et au début des années $1880^{\circ}$, tel N.K. Giers. Mais notons que si dans les années 1856-1857 le projet d'un gouvernement d'union excluait encore les questions de politique étrangère au sein du gouvernement, ici ce furent justement les hauts fonctionnaires du MID qui réintroduisirent ces idées, en insistant sur le fait que la politique extérieure ne pouvait pas être isolée des questions intérieures de la Russie. Ils demandaient donc à réformer le système et le ministère dans les mêmes termes.

En effet, l'appareil diplomatique russe s'était métamorphosé très lentement au cours des siècles et ses diverses réorganisations n'avaient jamais posé la question de la place du diplomate et de son travail. Le système collégial de prise de décision que Pierre le Grand avait instauré officiellement en 1720 au sein du Collège des Affaires étrangères (Kollegija inostrannyh Del) ne vit jamais le jour dans la mesure où il se présentait comme un moyen pour neutraliser les différents acteurs engagés dans cette prise de décision politique, laissant les mains libres à l'empereur pour mener sa propre politique. En 1802, Alexandre I n'eut qu'à institutionnaliser un système centralisé depuis plusieurs dizaines d'années en organisant les ministères en Russie, dont celui des Affaires étrangères.

Ensuite, au cours du XIX siècle, la structure du MID se figea, rendant encore plus difficile l'instauration d'une réforme, qui n'allait voir le jour qu'au début du $\mathrm{XX}^{\mathrm{e}}$ siècle. Mais la véritable spécificité russe résidait dans son organisation gouvernementale - centrée autour de la figure du tsar qui détenait la prérogative en matière de politique étrangère - dont dépendaient la conception et l'élaboration de la politique extérieure. Le ministère des Affaires étrangères était à la fois le lieu où s'élaborait la politique étrangère et l'outil de sa mise en œuvre : une telle organisation posait le problème du débat politique en Russie. Il n'existait pas de coordination de

6. V. G. Černuha, Vnutrennjaja politika carizma s serediny 50-h do načala 80-h gg XIX V. [Politique intérieure du tsarisme du milieu des années 1850 au début des années 1880], L., 1978, p. 136-198. Nous voulons nous servir de cette occasion pour faire remarquer que l'idée de United Government avancée par D. Mc Donald pour les années 1905-1906 n'est pas de lui. Le grand mérite de V. G. Černuha fut de mettre pour la première fois en lumière les débats qui se déroulèrent en 1856-1857, et surtout de réintroduire cette expression, qui n'est par ailleurs pas d'elle, mais comme elle le montre, de P. A. Valuev.

7. Ibidem, p. 77.

8. Ibidem, p. 71.

9. Ibidem, p. 119. 
travail entre la politique étrangère et la politique intérieure russes, entre les ministères, les organes gouvernementaux et la presse, sans parler des départements politiques du MID (la Chancellerie, le Département asiatique, le Conseil du ministère). Au moment de la mise en place de la réforme du MID, le regroupement des départements politiques en un seul fut une des idées centrales de la réorganisation : désormais, le diplomate ne voulait plus apparaître dépendant de la politique étrangère, il voulait participer à son élaboration.

Une des caractéristiques de la réforme du ministère des Affaires étrangères est qu'elle fut initiée par des diplomates qui avaient passé une grande partie de leur carrière dans les représentations diplomatiques à l'étranger. Ils arrivaient aux postes à responsabilité au MID au moment où la politique étrangère prenait de plus en plus d'importance au sein de la politique intérieure russe. Leur appréhension du politique les amena à accroître leur rôle politique sur le terrain, mais aussi dans leur travail au ministère. La progression de leur carrière influait désormais sur leur statut, qui dépendait dès lors de leur position dans l'administration, contrairement à la période antérieure, où leur position dépendait de leur statut : à cette même époque, on assistait, comme dans d'autres ministères, à une professionnalisation de la fonction de diplomate. Nous ne voyons pas de déclin de la noblesse dans la haute bureaucratie du MID : la domination de l'élite traditionnelle était encore plus ou moins constante. Mais elle avait perdu ses caractéristiques principales, à savoir la possession foncière, et devenait plus hétérogène. Ces hommes représentaient une élite sociale formant une classe de bureaucrates professionnels parce qu'elle avait dû s'adapter en tant que classe aux changements politiques et économiques survenus en Russie.

Ce fut Aleksandr Izvol'skij qui mit en place la réforme du MID. Le 28 avril 1906, il fut nommé ministre des Affaires étrangères, au moment de l'ouverture de la Première Douma. Il succédait au comte Lamzdorf. A. A. Savinskij, directeur de la Chancellerie du ministère depuis 1905, explique sa nomination à la tête du MID par la situation de politique intérieure de la Russie et par le rôle qu'Aleksandr Izvol'skij y aurait joué : il prit part aux pourparlers avec le parti des Cadets (KD), qui étaient partisans d'une extension des pouvoirs de la Douma ${ }^{10}$. Par ses origines, il se trouvait lié au milieu de la noblesse provinciale et des zemstva ${ }^{11}$. Il partageait les vues de ceux qui voyaient dans le libéralisme modéré le moyen de progresser vers des formes constitutionnelles de gouvernement.

À la nomination d'Izvol'skij, sur le plan intérieur, la Russie, après sa défaite contre le Japon, devait faire face à la mise en place d'une monarchie constitutionnelle dans laquelle le tsar continuait à diriger la politique étrangère. Le Conseil des ministres, à la tête duquel fut nommé le comte Witte, coordonnait la politique gouvernementale. Le ministre des Affaires étrangères, quant à lui, devait faire appliquer cette politique à l'étranger. Izvol’skij ne voulait pas se poser en exécutant

10. Cité par Valentin Alekseevič Emec, « A.P. Izvol'skij, ministr-neudačnik ili reformator ? » [A. P. Izvol'skij, ministre malchanceux ou réformateur ?], Novaja i novejšaja istorija, $\mathrm{n}^{\circ} 1$, 1993 , p. 33.

11. Mémoires d'Alexandre Iswolsky, ancien ambassadeur de Russie à Paris (1906-1910), P. : Payot, 1923, p. 93. 
de cette politique, mais bien en être l'un des initiateurs. Il pensait que pour retrouver sa position de grande puissance, la Russie devait à la fois régler ses problèmes sur le plan interne et externe. En sa qualité de ministre des Affaires étrangères, il allait s'attacher à moderniser son ministère. Sur le plan de la politique étrangère, il voulait tenter de redéfinir les priorités de la Russie et souhaitait préserver la Russie d'une guerre pour une durée de dix ans. La réforme du ministère des Affaires étrangères qu'il amorça répondait directement à ces nouvelles préoccupations, que ce fût sur le plan organisationnel ou structurel. C'est dans cette perspective qu'il faut comprendre l'importance qu'Izvol'skij accorda à la Douma et à l'opinion publique. D'après les Lois fondamentales, la Douma n'avait aucun contrôle sur la politique étrangère. Elle n'était qu'un des intervenants dans l'élaboration de cette politique. Mais elle allait devenir un des éléments les plus importants de la propagande en matière de politique étrangère par voie de presse. Izvol'skij mit en place une nouvelle approche dans les rapports entre la presse et le travail diplomatique.

En 1907, Izvol'skij plaça en étroit contact l'Agence télégraphique de SaintPétersbourg et la deuxième Expédition des journaux (Vtoraja Gazetnaja Ekspedicija), au sein du ministère, nommant la même personne, N.K. Giers, à la tête de ces deux institutions. Il allait occuper ces deux postes jusqu'en 1910, date qui coïncide avec celle de la fin du mandat ministériel d'Izvol'skij. Giers était un diplomate de carrière, carrière passée en grande partie à l'étranger; il avait les mêmes vues qu'Izvol'skij sur le rôle du ministre des Affaires étrangères et, plus largement, sur la prise de décision en matière de politique étrangère. Il diffusa une note au moment de la nomination d'Izvol'skij qui illustre très bien les nouvelles préoccupations politiques qui agitaient les hauts fonctionnaires russes : c'était une critique acerbe du ministère des Affaires étrangères russe, plus particulièrement de son rouage central, la Chancellerie du ministère ${ }^{12}$. L'auteur préconisait une concertation dans l'action politique et accordait une importance considérable à la Douma, par la voix des « représentants du peuple». Le tsar n'était donc plus le seul décideur ${ }^{13}$. La critique portait essentiellement sur l'absence de programme défini en matière de politique étrangère, à cause de l'administration même du MID qui ne faisait pas de distinction entre la politique intérieure et la politique extérieure.

Izvol'skij considérant qu'ils devaient travailler en étroite collaboration, les relations entre l'Agence télégraphique et le ministère des Affaires étrangères ne cessèrent de croître pendant ces années. Ainsi, c'est sur les ordres du MID que les bulletins concernant la politique étrangère devaient être approuvés par le ministre ou par

12. Cette note tirée à une cinquantaine d'exemplaires n'était pas destinée aux journaux, mais à un public restreint de hauts fonctionnaires du pays en poste dans les plus hautes instances gouvernementales et politiques, et plus précisément au nouveau ministre des Affaires étrangères, Izvol’skij. Cité par V.A. Emec, «Zapiska N.K. Girsa o dejatel'nosti MID Rossii », Isledovanija po istočnikovedeniju istorii Rossii (do 1917), M., 1993, p. 188.

13. Cette nouvelle prise de décision était la conséquence des nouvelles formes gouvernementales, apparues après la Révolution de 1905. Ainsi, au sein du Conseil des ministres étaient débattues les questions concernant les relations de la Russie avec les gouvernements étrangers, et en particulier, les divers accords commerciaux. 
un organe accrédité par le MID. En effet, le MID vérifiait, avant leur publication, toutes les informations susceptibles de toucher au domaine de la politique étrangère. La collaboration entre l'Agence télégraphique de Saint-Pétersbourg et le ministère des Affaires étrangères fut élaborée sur le modèle des rapports qu'entretenaient le ministère des Finances (dirigé jusqu'en 1903 par le comte Witte) et l'Agence commerciale télégraphique (Torgovo-telegrafnoe agenstvo). Cette agence était en contact direct avec les correspondants du ministère des Finances à l'étranger. Ces derniers travaillaient dans les représentations russes à l'étranger, mais dépendaient directement du ministre des Finances.

Par ailleurs, toutes les affaires politiques furent centralisées en un seul département, évitant ainsi la création d'une sorte de ministère des Affaires étrangères concurrent et indépendant, comme le Comité d'Extrême-Orient créé en 1903, et qui avait conduit au conflit russo-japonais. La Chancellerie du ministre devint la Chancellerie privée du ministère, à l'image du Cabinet du ministre à l'étranger ${ }^{14}$. Ainsi, le département politique était confié à une seule personne ${ }^{15}$, le premier adjoint du ministre des Affaires étrangères, chargé de gérer quatre sections politiques. Chacune d'elle recouvrait une zone géographique précise : la section de l'Europe, de l'Amérique et de l'Afrique, la section du Proche-Orient, incluant l'Abyssinie et l'Egypte, la section de la Perse et de l'Asie Centrale et enfin celle de l'ExtrêmeOrient. Des archives furent instaurées auprès du département politique, ainsi qu'un département de lithographie, et une section juridico-consulaire, à la tête de laquelle avait été nommé le second adjoint du ministre ${ }^{16}$.

Izvol'skij cherchait donc à donner du sens à la politique étrangère, en répartissant les sections politiques, d'une part, par aires géographiques, et d'autre part, par problèmes politiques ${ }^{17}$. Le rôle du ministère fut redéfini. Par rapport aux statuts de 1868, mis en place par Gorčakov, le changement du rôle du ministère se traduisait par la prise en compte par le MID de tous les aspects d'un pays, sur le plan juridique, économique, financier, commercial, etc., et non plus simplement sur les aspects politiques, qui ne concernaient que les relations que la Russie entretenait avec ces pays. En effet, le département politique s'occupait en son sein à la fois de questions politiques et dans sa section juridico-consulaire, de questions traitant des aspects juridiques, économiques, financiers qui pouvaient revêtir une importance politique.

Cette redéfinition du rôle du MID s'inscrivait, à notre avis, dans le changement politique qui était survenu dans le pays après 1905 , avec le choc produit par la défaite russe au Japon, et la mise en place d'une monarchie semi-constitutionnelle

14. AVPRI, f ; 159, op. 731, d. 60,1.5. Journal de la deuxième réunion de la commission créée au MID en vue de la réforme des institutions centrales du Ministère, le 7 juin 1907.

15. Ibidem, 1. 5 .

16. AVPRI, f. 159, op. 731, d. 67, 1. 4. Journal de la deuxième réunion de la commission créée au MID en vue de la réforme des institutions centrales du ministère, le 18 juin 1907.

17. AVPRI, f. 159, op. 731, d. 60,1. 107. Les questions à débattre au sein de la Commission en vue de la réorganisation du MID. 
qui permit de modifier le mode de prise de décision en matière de politique étrangère, aussi bien sur le plan gouvernemental qu'au sein du ministère lui-même. Parallèlement à cette réorganisation structurelle du ministère, était discutée la formation du diplomate. Pour les fonctionnaires qui se destinaient au travail à l'étranger, il était envisagé qu'ils fassent un stage, avant leur départ, dans tous les départements du MID, ainsi que dans le département consulaire. Et, pour plus de mobilité, ils devaient changer de mission tous les trois ans. Cette rotation leur aurait permis à terme de se familiariser avec tous les aspects de la vie diplomatique ${ }^{18}$. Ainsi, l'apprentissage du métier de diplomate ne s'achevait plus avec l'examen, mais se prolongeait tout au long de sa carrière.

Il en ressort qu'à la fin du XIX et au début du XX $X^{\mathrm{e}}$ siècle, contrairement à l'image statique souvent présentée, le ministère des Affaires étrangères fut une administration qui tenta de sortir d'un immobilisme datant pratiquement de sa création, au début du XIX siècle. Un certain nombre de facteurs nourrirent la réflexion des diplomates russes dès la fin du XIX ${ }^{\mathrm{e}}$ siècle. Qu'il s'agisse de la guerre russo-japonaise sur le plan international, du traumatisme subi lors de la révolution de 1905 sur le plan intérieur, révolution par ailleurs annoncée par certains diplomates, ou de l'arrivée d'une nouvelle génération d'hommes, de la prise en compte de nouveaux paramètres d'ordre financier, commercial ou juridique, dans la politique étrangère, tout ceci permit de mûrir un projet de restructuration du MID qui aurait dû exercer une influence certaine sur le travail du diplomate.

Malheureusement cette réforme, victime entre autres des lenteurs administratives de la Commission budgétaire, ne fut soumise à la Douma qu'en juillet 1914, au moment où la Première Guerre mondiale éclatait et, bien qu'acceptée par la Douma, elle ne fut jamais mise en œuvre.

Centre franco-russe de sciences sociales à Moscou/EHESS

mrakuzin@yahoo.fr

18. Correspondance de P.S. Botkin avec A.P. Izvol'skij (1906-1907), Diplomatičeskij ežegodnik, 1990-1992, Lettre n 4, 18 janvier 1907, p. 292-295. 